\title{
Non-locality by nanoconfinement
}

Coherent ultrafast spectroscopy of nanofocused plasmonic pulses evidences a nonlinear optical phenomenon driven by the extra momentum available at the nanoscale and controlled by the Doppler effect.

\section{Andrea Giugni}

Current nanotechnology research in optoelectronics and nonlinear optics has heavily targeted twodimensional materials (2D), because they possess appealing electronic, optical, and plasmonic properties that, in many cases, can be conveniently tuned by external fields. These properties arise from the unconventional 2D atomic structure and quantum confinement effects of these materials, even though a rigorous theoretical explanation still remains elusive.

Atomic thick graphene material, in particular, possesses a large third-order nonlinear coefficient, thanks to the high Fermi velocity of massless Dirac electrons in its gapless 2D band structure [1]. However, the nonlinear response remains too weak for practical purposes because the intrinsic nonlinear susceptibility is still too small to support efficient light-controlled-by-light operability. An efficient switching would require absorption changes as large as $90 \%$ or equivalently a nonlinear phase shift about 180 degrees in a distance not significantly larger than the absorption length scale. However, this has not been possible to achieve in small volumes. Nevertheless, many 2D materials, and graphene, in particular, appear to be our best candidates for an externally enhanced third-order coefficient material, due to their exclusive blend of physical properties, and to the possible strong interaction of localized plasmonic fields with the whole electronic cloud. This effect has been recently shown using large field gradients from tapered nanostructures[2, 3]. Now Jiang et al. have been able to enhance the third-order optical response in one to a few layers graphene using nanoscopic nonlinear near-field excitation[4]. The enhancement is a combination of the large momentum available in the plasmonic near-field and a Doppler shift broadening. Their images show clear contrast and high signal-to-noise intensity ratio in single to few layers graphene, revealing unusual nonlocal effects.

Nonlinear optical imaging requires broadband, femtosecond temporal, and nanometric spatial control of a synchronized pair of ultrafast optical pulses[3], aside from an efficient way to guide and concentrate far-field radiation well below the diffraction limit. The most effective way to do nanoimaging is to excite surface plasmon polaritons (SPPs) in a tapered metallic structure ending in a nanometric apex. The excited SPPs propagate at the surface of the tapered metal and adiabatically focus at the tip's apex[5] with a mechanism that is intrinsically broadband, ideal for the control of ultrafast plasmonic pulses in the time domain. The SPP field intensity can easily exceed an enhancement of $10^{3}$ at the apex in a spot comparable to the radius of curvature, $R$, of few nanometers, thus enhancing local scattering phenomena manifolds. A chirped optical grating designed on the metal surface accomplishes the momentum mismatch between the two energy carriers launching ultrafast plasmonic pulses toward the apex with good efficiency (Fig. 1a). Structural chemical sensing[6], nano coherent Raman[7], and hot-electron transport and imaging at 
the nanoscale[8], have been already demonstrated with comparable plasmonic tools, proving a mature and versatile approach to nanoscale investigation.

Using a similar setup, Jiang et al. have succeeded in nanoimaging graphene using nonlinear optical four-wave mixing (FWM), a third-order interaction $\left(\omega_{\mathrm{FWM}}=2 \omega_{1}-\omega_{2}\right)$ between two femtosecond pulses ( $\omega_{1}$ and $\omega_{2}$ ) locally excited by the intense near-field confined at the apex of the tapered structure. The possibility to enhance this third-order effect allows the researchers to use it for imaging and spectroscopic purposes.

a

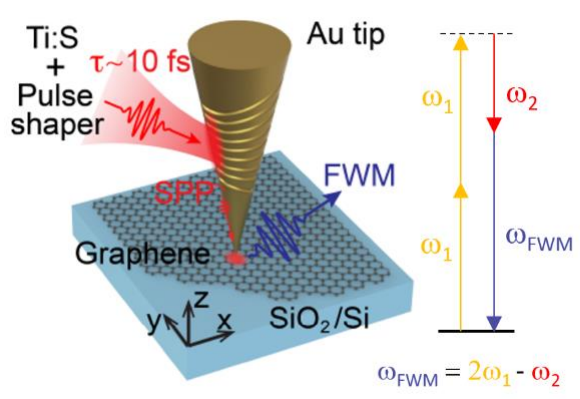

b

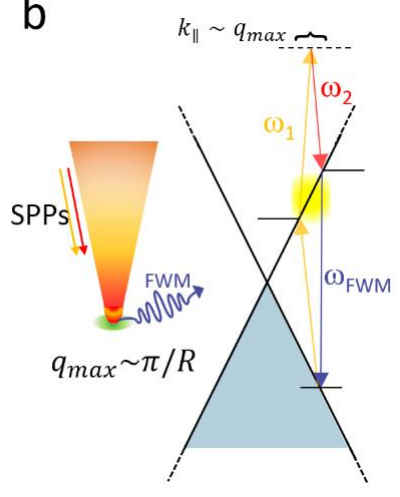

C

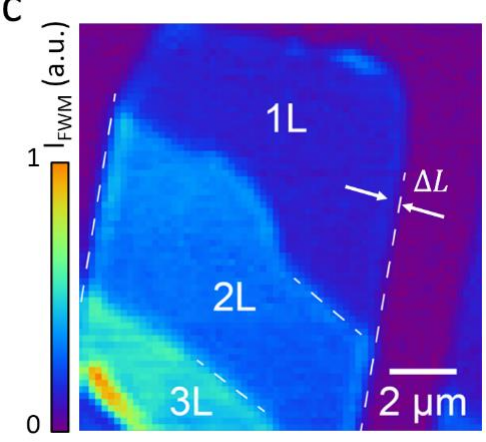

\section{Caption}

Fig. 1 I Ultrafast nano-optical imaging in graphene. a Experimental layout for the nano-localized FWM excitation. A Au plasmonic cone with a chirped grating-coupler adiabatically nanofocuses two femtosecond pulses at frequency $\omega_{1}, \omega_{2}$ at the tip apex transferring the nano-localized excitation in the graphene sample. Graphene emits the FWM signal, $\omega_{F W M}$, at $2 \omega_{1}-\omega_{2}$ as sketched in the energy conservation scheme. b SPPs momentum $q_{\max }$, Dirac cone, and $2 \omega_{1}-\omega_{2}$ FWM process resonantly driven by large in-plane near-field momentum $k_{\|}$from the tip. The supplied wavevector compensates the momentum difference of nonresonant transitions. $\mathrm{c}$ Near-field FWM imaging of single and few-layer graphene. The dashed white line indicates actual graphene edge. Arrows quantify the modelled FWM edge-enhanced spatial delocalization $\Delta L \sim 2 \pi v_{F} / \Delta \omega_{F W M}$. (a) and (c) partially reproduced from ref. 4.

The gapless linear dispersion between energy and momentum of electrons in graphene is responsible for a spectrally flat absorption ( $2.3 \%$ for the single atomic layer) and the strong thirdand higher-order optical responses. In particular, the large $\chi^{(3)}$ nonlinearity, about two orders of magnitude higher than the ones for comparable gold films, arises from the vertical transition at frequencies of $\omega_{F w M}, \omega_{1}$, and $\omega_{2}$, all equally resonant. This feature has motivated numerous quantum nonlinear optic and ultrafast dynamic investigations[9], whereas the use of localized plasmonic devices and quantum confinement in nanostructures have been proposed to enhance the graphene nonlinear signal. However, with the imaging technique proposed by Jiang et al. it is now possible to investigate coherence, dephasing time, and optical nonlinearities in the material exploiting a nanoscopic excitation as small as $\pi R^{2}\left(<10^{4}\right.$ atoms), their properties, and their dependence on edges, layers, folds, and other finite-size effects. The locally excited FWM in graphene, promoted by a combination of symmetry breaking and retardation effects at the tip, is resonantly originated by the large plasmonic momentum, available up to $\mathrm{q}_{\max } \approx 2 \pi / \mathrm{R}$ (Fig. $1 \mathrm{~b}$ ), and enhanced by a polarization sensitive effect in the vicinity of edges and structural heterogeneities that further lift the degeneracy. The phenomenon is qualitatively different from the intrapulse FWM from the Au tip 
(generated by the plasmonic strong near-field gradient), and from the far-field excitation (momentum conservation). The relaxation of the classical momentum conservation at the tip causes the FWM enhancement as a non-local FWM emission, allowing the Doppler effect identification and characterization.

Jiang et al. also extend the third-order nonlinear perturbation theory for graphene and quantify the effect of the high near-field momentum available on the spatial dispersion. The identified Doppler shift broadening in the third-order density matrix element for the electronic transition combines the high electron Fermi velocity in graphene with the high momenta of the tip apex field, introducing a nonlocal enhancement in the FWM signal within a characteristic delocalization, which shows a remarkable agreement with experimental observations (Fig. 1c).

Besides graphene, a large class of layered material in the form of van der Waals heterojunctions [10] and composite films shows size-dependent bandgaps and plasmon frequencies [11]. Doppler effects on the nonlinear response are also expected for all those 2D materials with high group velocity electrons. The technique developed by Jiang et al. can now be used to probe nanoscale electronic phenomena at the time scales of electronic dephasing in other layered materials. The richness of information gathered by a multi-spectroscopy nanoprobe approach can unveil the relations between functional and structural properties of matter at the spatial and temporal scales of quantum effects, thus supporting fundamental studies of light-matter interactions for integrated optoelectronics, and nanophotonics devices. In perspective, it would be possible to extend the technique to the generation of surface plasmons at $\mathrm{THz}$ frequencies through tip-enhanced difference-frequency generation process, or with a sufficient bandwidth probe coherently excited vibrational modes in implementation of ultrafast coherent anti-Stokes Raman spectroscopy.

Andrea Giugni ${ }^{1}$

${ }^{1}$ King Abdullah University of Science and Technology (KAUST), Physical Science and Engineering Division (PSE), SMILEs Lab, Thuwal 23955-6900, Saudi Arabia.

Email: andrea.giugni@kaust.edu.sa

\section{References}

[1] F. Bonaccorso, Z. Sun, T. Hasan, A.C. Ferrari, Graphene photonics and optoelectronics, Nature Photonics, 4 (2010) 611.

[2] V. Kravtsov, S. AlMutairi, R. Ulbricht, A.R. Kutayiah, A. Belyanin, M.B. Raschke, Enhanced ThirdOrder Optical Nonlinearity Driven by Surface-Plasmon Field Gradients, Physical Review Letters, 120 (2018) 203903.

[3] V. Kravtsov, R. Ulbricht, J.M. Atkin, M.B. Raschke, Plasmonic nanofocused four-wave mixing for femtosecond near-field imaging, Nature nanotechnology, 11 (2016) 459.

[4] T. Jiang, V. Kravtsov, M. Tokman, A. Belyanin, M.B. Raschke, Ultrafast coherent nonlinear nanooptics and nanoimaging of graphene, Nature nanotechnology, DOI 10.1038/s41565-019-0515$x(2019)$.

[5] M. I. Stockman, Nanofocusing of Optical Energy in Tapered Plasmonic Waveguides, Physical review letters, 93 (2004) 137404. 
[6] F. De Angelis, G. Das, P. Candeloro, M. Patrini, M. Galli, A. Bek, M. Lazzarino, I. Maksymov, C. Liberale, L.C. Andreani, E. Di Fabrizio, Nanoscale chemical mapping using three-dimensional adiabatic compression of surface plasmon polaritons, Nat Nano, 5 (2010) 67-72.

[7] S. Berweger, J.M. Atkin, R.L. Olmon, M.B. Raschke, Light on the Tip of a Needle: Plasmonic Nanofocusing for Spectroscopy on the Nanoscale, The Journal of Physical Chemistry Letters, 3 (2012) 945-952.

[8] A. Giugni, B. Torre, A. Toma, M. Francardi, M. Malerba, A. Alabastri, R.P. Zaccaria, M.I. Stockman, E. Di Fabrizio, Hot-electron nanoscopy using adiabatic compression of surface plasmons, Nature nanotechnology, 8 (2013) 845-852.

[9] H.K. Kelardeh, V. Apalkov, M.I. Stockman, Graphene in ultrafast and superstrong laser fields, Physical Review B, 91 (2015) 045439.

[10] A. Giugni, B. Torre, M. Allione, G. Das, Z. Wang, X. He, H.N. Alshareef, E. Di Fabrizio, Experimental Route to Scanning Probe Hot-Electron Nanoscopy (HENs) Applied to 2D Material, Advanced Optical Materials, 5 (2017).

[11] D.B. Velusamy, J.K. El-Demellawi, A.M. El-Zohry, A. Giugni, S. Lopatin, M.N. Hedhili, A.E. Mansour, E. Di. Fabrizio, O.F. Mohammed, H.N. Alshareef, MXenes for Plasmonic Photodetection, Adv Mater, 0 (2019) e1807658. 